\title{
National Ocean Worlds Analog Test Facility and Field Station
}

A White Paper reflecting needs for outer planet sub-surface access technology and science, submitted to the Decadal Survey in Planetary Science and Astrobiology

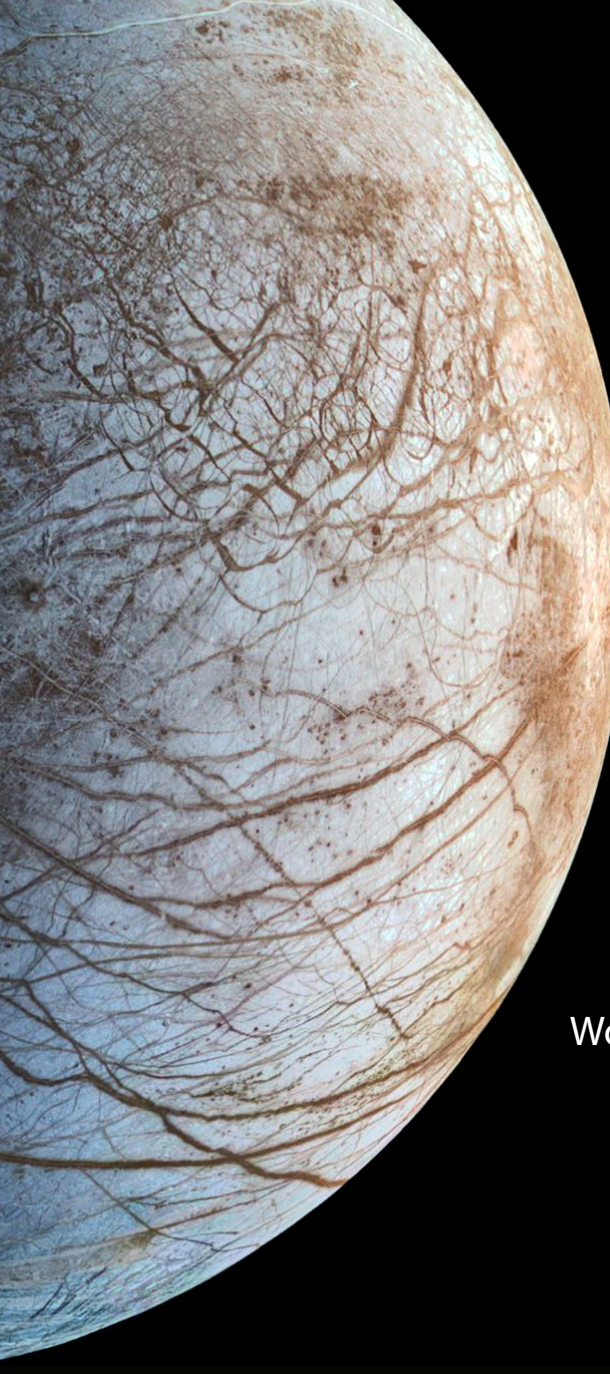

Lead Author: William C. Stone* Stone Aerospace

Co-Authors:

Samuel M. Howell NASA Jet Propulsion Laboratory California Institute of Technology

Nathan Bramall Leiden Measurement Technology

Chris German

Woods Hole Oceanographic Institution

Alison Murray

Desert Research Institute

Vickie Siegel

Stone Aerospace

*Contact: billstone@stoneaerospace.com |

512.534.8759

Signatories and Participants in the November 4, 2019 Europa Station Workshop held in Austin, Texas: Dan Berisford (JPL); Don Blankenship (UTIG), Brent Christner (University of Florida), Kate Craft (JHU-APL); Tom Cwik (JPL); Peter Doran (Louisiana State University), Jamin Greenbaum (UTIG); Kevin Hand (JPL), Bart Hogan (Stone Aerospace); Boleslaw Mellerowicz (Honeybee Robotics); Joseph Palmowski (Honeybee Robotics); Kristof Richmond (Stone Aerospace); Mark Skidmore (Montana State University), Miles Smith (JPL); Dale Winebrenner (University of Washington APL); Natalie Wolfenbarger (UTIG).

A list of additional individuals who have endorsed this white paper can be found at: tinyurl/CRYOBOTS-YES 


\section{Summary}

We recommend the establishment of complementary full-time national Ocean World Stations Ocean World Field Station in Antarctica and Ocean World Simulator Station (a cryogenic-ice vacuum test facility within the continental United States) for advancing TRL of Ocean World subsurface and in situ mission technologies through analog testing. We further recommend sustained financial support for research and development of subsurface access systems, culminating in the use of these facilities to enable Technology Demonstration Missions of sub-ice access in Antarctica. We urge that NASA and NSF collaborate constructively to assure the successful implementation of the Ocean World Field Station.

\section{$\underline{\text { Science Justification }}$}

Many white papers submitted to the 2023-2032 Decadal Survey committee address the nature of Ocean Worlds and the critical scientific questions and rationales associated with their continued study. The paper submitted by the Network for Ocean Worlds (NOW), in particular, argues for a

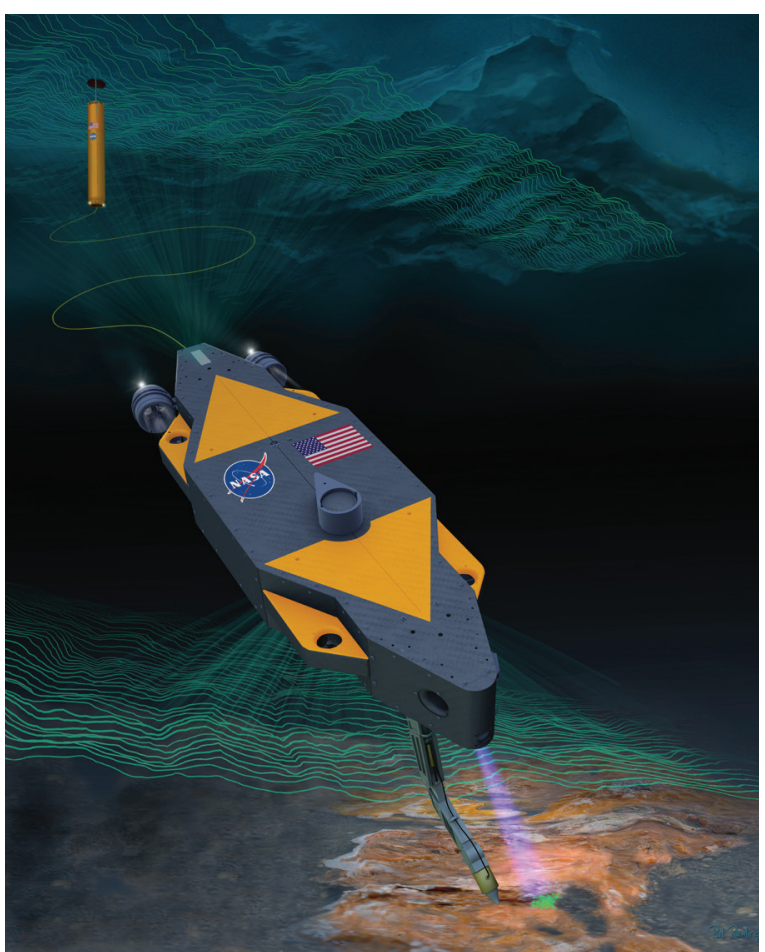

Figure 1: Artist's conception of an Antarctic subglacial access and life search mission involving a cryobot-deployed AUV. This concept aligns closely with possible future Ocean World sub-surface missions. Program of Ocean Worlds in which a first landed mission to an Ocean World should be prioritized in the coming decade, followed by a dedicated Ocean Access/Life Detection mission in the decade to follow [1]. To be poised to enable such future subsurface exploration of Ocean Worlds much needs to begin immediately in the way of technology development and, critically, in technology testing, assessment, and iterative design in analog environments. The degree of autonomy required for such sub-ice missions is significantly greater than that for current Mars rover missions due to the substantially greater dynamic in situ unknowns. The SNOW group within OPAG has submitted a white paper to this decadal committee regarding their assessment of the priority technologies needed to further Ocean World exploration [2]. The authors of this present paper advocate for the creation and sustained maintenance of testing facilities that must be created to meaningfully test and advance TRL for such technologies.

We recommend that two facilities should be established and made generally available to Ocean Worlds technology development groups for research, development, and testing. The first is a large scale, semi-permanent field camp established in either Greenland or Antarctica (but preferably the latter to take best advantage of existing support infrastructure), at a site which provides access to a subglacial lake that is overlain by multiple kilometers of ice. A parallel site may also be needed to test access and mobility through ice shelves to an active sub-surface ocean. The second facility is a domestic laboratory housing a silo of ice maintained under environmental conditions relevant to Ocean World surfaces, and near-surface ice conditions. 
During the past 20 years NASA has invested more than $\$ 50 \mathrm{M}$ in $\mathrm{R} \& \mathrm{D}$ in the areas of ice penetration technologies, sub-ice ocean exploration and science mobility platforms, together with specialized life detection instrumentation in its ASTEP, PSTAR, COLDTECH, ICEE2 and SESAME programs. This research has taken ideas that were considered science fiction in previous decades and turned them into functioning engineering prototypes that have demonstrated a clear path forward. For extensive, up-to-date, state-of-technology summaries for this technology, see references [3-7]. Detailed examples of several operational prototype cryobots are given in $[8,9]$ and a state-of-technology summary on mobile sub-ice autonomous science and exploration systems is presented in [10].

There are many concepts for both cryobots (ice penetrating robots) as well as sub-ice autonomous underwater mobility systems (Figure 2). Bringing them to TRL7 in a manner robust enough to survive a multi-year sub-ice mission (Figure 3) necessarily requires sustained analog environment testing. Creating such capabilities at the beginning of this decade, accessible to all researchers, will prove a fundamental underpinning of any successful Ocean Worlds Program.

Analog Testing Needs for Ocean World Missions

While the putative subsurface conditions of Ocean Worlds (Enceladus, Calisto, Ganymede, Triton, Titan, Europa) vary significantly, Europa provides a strong bounding example of the possible logistical challenges facing an ocean-access mission: an ice shell of unknown thickness, potentially ranging from 10 to 40 kilometers thick, overlaying an ocean up to 130 kilometers in depth, and covering a rocky core. The ice shell (and potentially the rocky interior) tidally flexes in response to the gravitational interactions with Jupiter, thus generating the heat to support the hypothesized liquid ocean (Figure 4). Within this context, there are at least six critical environments that all mobility platforms targeting a subsurface ocean must contend with:
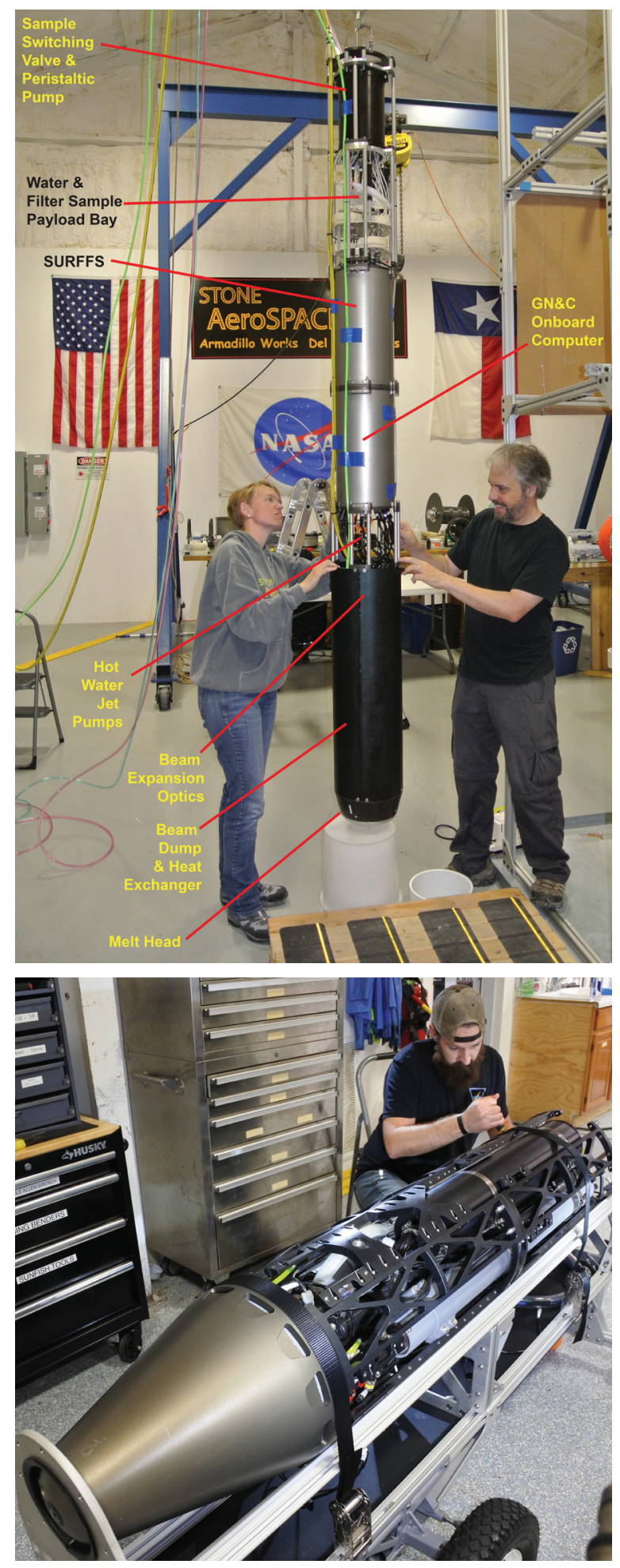

Figure 2: Two examples of NASA-funded operational cryobots: VALKYRIE (using a high power laser as a nuclear surrogate); and THOR, using a remote $10 \mathrm{kV} \mathrm{AC} \mathrm{power}$ source as a nuclear surrogate. 
1. The Starting Problem: An ice penetrator must transition from the ultra-high-vacuum, ultracold environment of a surface lander to starting and entering its borehole, through the inevitable borehole closure behind it. The surface of Europa has a mean temperature of $100 \mathrm{~K}$ and an ultra-high vacuum of $10^{-5}$ Torr. To mitigate this challenge, a variety of enabling start-up technologies to start at the surface have been proposed and now must be tested to raise TRL and credibility. However, there are no analogs for the Starting Problem on Earth. The surface conditions of icy, airless worlds must be created in the laboratory. To overcome the Starting Problem, the facility must be large enough to handle maximum-permissible-volume, flightlike subsurface access systems, maintain $100 \mathrm{~K}$ temperatures without boundary effects and $10^{-5}$ Torr, and be tall enough to allow several vehicle lengths of descent in order to establish hole clo-

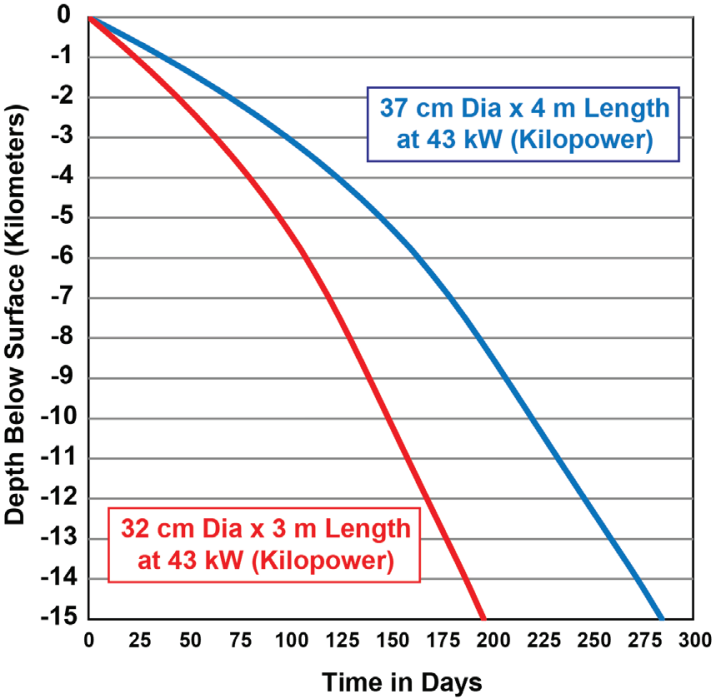

Figure 3: Example Ocean World performance curves for realistically sized cryobots using Kilopower thermal sources. Calculations account for melt efficiency, ice temperature vs depth, and vehicle speed effects [3]. sure and steady state descent behavior.

2. Cold Ice Cruise: Cryobot transit through cryogenic, potentially tectonically active ice (temperatures less than $180 \mathrm{~K}$ ). Ocean world ice shells are composed of cold, near-surface ice, that sustains fracturing and faulting, and warm interior ice that relaxes to prevent fracturing and may be convecting. Each candidate penetration technology will need to be tested with the vehicle completely encased in ice at the cryogenic temperatures found in the fractured region of Ocean World ice shells. For example, a mechanical boring system that would use moving machinery may be subject to unusual wear and fatigue patterns in ultra-low temperature ice; and, furthermore, ice cuttings, being less dense than the native ice, may eventually jam such a mechanical cutting system as there is no exit path for the cuttings. On the other hand, nuclearpowered passive melt probes and water jet drill systems are strongly dependent on the amount of heat dissipated through the side walls of a probe in cold ice. Development and testing of effective side-wall thermal management technologies will be critical to this type of system. All of these criteria argue for a national facility large enough to test full scale vehicles without boundary effects.

3. Active Guidance and Navigation: Areas requiring identification of obstacles within the ice, (e.g. sediments, salt intrusions) and identification of targets (e.g. brine layers, water-filled cracks). Following identification of such features the cryobot may be required to change course to avoid or, indeed, to encounter them. Lacking ground-truths, we can make predictions of the range of features that might be encountered by a descending cryobot, including: variations in the temperature of the ice, variations in impurities in the ice (including salts and other chemical compounds), the possible presence of dust and other impact fragments, the possible presence of salt or debris inclusions; and the possible presence of gas-filled and liquid water-filled voids prior to reaching the ocean. We collectively define these as "obstacles", though under some cir- 
cumstances they may represent important targets in the search for evidence of life. Each of these possibilities can and should be included in laboratory and field testing and evaluation of all potential cryobot architectures.

4. Warm Ice Cruise: Cryobot transit through more temperate ice (temperatures above $180 \mathrm{~K}$ ) with different physical characteristics. As a cryobot approaches warmer ice close to the liquid ocean, the ice now flows in solid state to close any cracks, but new challenges arise. In addition, the ice becomes more insulating and the vehicle must respond with appropriate thermal management. This phase may represent the majority of the descent depth to the subsurface ocean, but the minority of transit time, with temperature profiles and ice constituency that will be similar to deep ice over subglacial lakes on Earth.

5. Breakthrough: Cryobot transit through the transition from solid ice travel into the liquid ocean. Two situations can arise in which a cryobot may find itself in freefall: en- Figure 4: Stages of sub-surface countering a) a vacuum-, gas-, or liquid-filled void in the Ocean World descent: 1) Startice column; and b) entry at the sub-surface ocean. In both ing Problem; 2) Cold Ice Cruise; cases, the same design requirement emerges: the cryobot 3) Active Guidance and Obstacle must identify imminent or in-progress breakthrough situ- Avoidance; 4) Warm Ice Cruise; 5) ations and maintain vertical control through the event to Ocean Breakthrough; and 6) Ocean avoid a premature termination of the mission. A vertical Exploration. Modified from Howellmotion control system, as well as a co-dependent com- Pappalardo 2020 [11].

munications link to the lander, can be effectively evaluated at on Earth. Simulating these phases in a subglacial lake environment allows assessing behavior at the interface when the vehicle must control descent. Mechanical drilling systems designed for cold ice may have problems with binding in this zone and tests of remedial measures can effectively be performed at a terrestrial polar facility.

6. Ocean Exploration: Moving through the ocean to explore, characterize, and sample in the search for life. While a dedicated cryobot subsurface mission may be the extent to which a national program aspires in the 2033-2042 timeframe, it is important to understand that any life in a low energy environment may be concentrated around energy sources (e.g. at seafloor hydrothermal vents, or areas of ice downwelling). The probability of encountering such a location on Earth by lowering an instrument sonde from a research vessel from any random point on the surface is vanishingly small. It will be no different on Ocean Worlds. To address this, the exploration radius must be significantly increased from the essentially one-dimensional mission profile of the cryobot. Thus, an advanced program to develop and analog test cryobotdeployed AUVs is needed to enhance the probability of the detection of life. 


\section{Description of Primary Facility Needs}

Of the six environmental regimes described above for a subsurface Ocean Worlds mission, the first two must be created artificially on Earth. The remaining four can be effectively tested in deep polar environments on Earth. We argue that the most effective location to pursue this is likely to be in Antarctica, leveraging existing logistics support as well as the diversity of already-characterized subglacial water bodies there, providing access to a wide range of possible energy environments. The general characteristics of both Ocean World Stations' requirements are described below. The exact specifications and locations for each facility should be determined by community consensus as part of the broader Ocean Worlds Program. It is the pressing need for their existence that we argue herein.

\section{Ocean World Simulator Station}

While NASA possesses numerous thermal-vacuum chambers for spacecraft testing, none of these facilities simulate Ocean World ice shells in the robust, systematic way needed to support subsurface systems development. Currently, there is only one operational "cryo-vac" facility in the United States dedicated to the testing of cryobots under environmental regimes 1) and 2) listed above, and that is a sub-scale chamber operated by industry (Figure 5). Until recently, the necessary size of a national facility had not been fully considered, but recent thermal analyses and test results from the sub-scale cryovac chamber suggest that the minimum diameter for such a facility is on the order of 20 vehicle diameters to prevent boundary effects compromising the thermal properties of the ice around and ahead of a descending cryobot. Similarly, because hole closure behind a descending cryobot typically occurs over a distance of approximately two or more vehicle lengths and because thermal boundary effects over the length of the vehicle are highly non-linear, if one is to accurately measure terminal velocity, the depth of the ice column should be a minimum of 3 to 5 vehicle lengths. In the bounding case, a cryobot designed to accommodate a $50 \mathrm{~kW}_{\text {th }}$ micro fission reactor as its heat source (e.g. the NASA-GRC Kilopower reactor that was successfully hot fired at LANL in 2019) will be between 30-40 cm diameter. With all necessary internal systems such a vehicle could be on the order of 6 $\mathrm{m}$ or more in height. Overall, these criteria indicate that a "national" level facility is needed with an ice column diameter in the range of $10 \mathrm{~m}$ and $30-50 \mathrm{~m}$ tall. Such a facility must be designed to support ice temperatures between 38 to $100 \mathrm{~K}$, and surface pressures varying from hard vacuum to $1.45 \mathrm{bar}$, and maintaining these while a test vehicle is injecting up to $50 \mathrm{~kW}_{\text {th }}$ into the ice.

\section{Ocean World Field Station: A Polar Regions Analog Environment}

As Figure 3 indicates, descent through a 15 kilometer thick ice cap with a cryobot could entail a 6 to 12 month or more mission. It will be critical to test vehicle concepts in an environment where descent depths and times are significant. Access to low energy subglacial environments will be needed to validate life detection instruments and decisions made autonomously. Bi-directional cryobots may need to

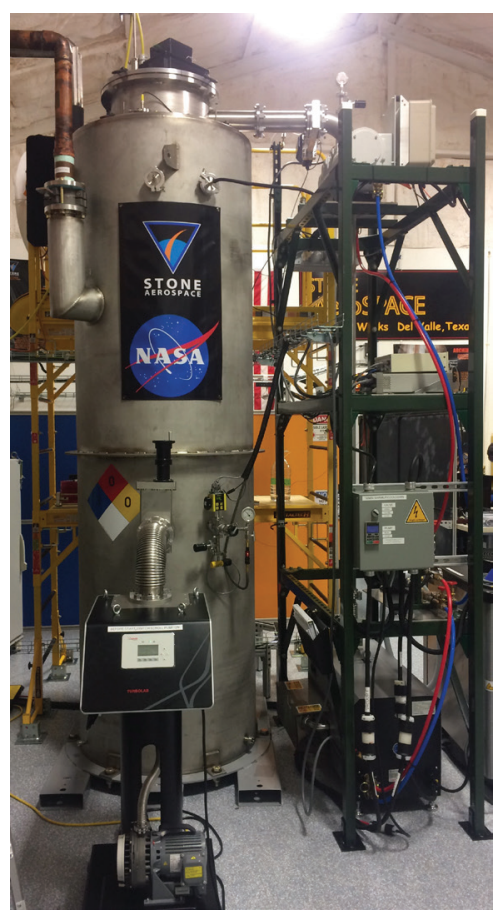

Figure 5: The Europa Tower cryovac facility was designed specifically for subscale cryobot testing. 
be developed to enable routine scientific access to subglacial lakes and importantly recovery of samples collected by automated science payloads for subsequent laboratory ground truth analysis. These capabilities remain notional, and to date only three isolated, short duration (a few hours to days) entries have ever been made into Antarctic subglacial lakes, using massive traditional and hot water drilling infrastructure. Ocean World Field Station will make such access routine by replacing these with self-contained cryobots.

The Antarctic ice sheet overlaying unexplored sub-glacial lakes represents the closest analog environment we have on Earth for testing advanced Ocean World missions. The $4 \mathrm{~km}$ thickness of Antarctic ice above a subglacial lake can be viewed as the last $4 \mathrm{~km}$ of relatively warm ice on an Ocean World before ocean breakthrough. As such, the ice sheet provides an extraordinary opportunity for full mission simulations, testing of life search protocols and algorithms, and determining the reliability of various concepts when subjected to long-term operations and exposure such as will be experienced on multi-year missions to an Ocean World. We envision Ocean World Station to be located in the Antarctic interior at a location where a minimum of 2,500 $\mathrm{m}$ and up to 4,000 $\mathrm{m}$ of ice overlies a suitable sub-glacial lake. The lake would be chosen, by community consensus, to have high likelihood of exhibiting certain features providing key astrobiological relevance to Ocean World waters: low overall energy flux that will serve as an analog to an outer planet subsurface ocean, with potential for localized energy sources such as hydrothermal vents that may be used to test instruments and methods for autonomous life search, identification, and characterization.

We stress the need for long duration sub-ice mission deployments on the order of months to years, not hours as is presently the case with drilled-access scientific programs in Antarctica - where access holes freeze shut within 24 to 36 hours. Methods will need to be developed and tested to routinely penetrate the ice with a cryobot, to deploy a mobile sub-ice AUV on the scale of months to years, to maintain communications and relay data to the surface, and to recover the entire system to the surface at the conclusion of a mission. This last capability will be critical to enable detailed analysis of vehicle sub-systems, as well as retrieving ground-truth biological or geochemical specimens and samples that may be obtained by both cryobots and AUVs.

In addition, though current discussions within NASA postpone a combined cryobot-AUV mission to some unspecified date, the level of AUV development required to accommodate the size, power, communications and other limitations set by the practicalities of cryobot deployment is significantly greater than that required for a successful cryobot-only mission. This implies starting deployable sub-ice AUV technology development immediately, even if a launch window will be several years beyond that for a cryobot-only mission. Such a mission is on par with the 3-decade span of Mars rovers leading to the present-day MSR Mission-suite.

Why NASA and NSF must Actively Collaborate

We wish to address frankly here the current reality of NASA's access to the Antarctic. By U.S. law, all scientific access to Antarctica involving U.S. federally-funded research must proceed through the National Science Foundation (NSF). NASA's Planetary Science and Technology through Analog Research (PSTAR) program has been one of the primary avenues for funding Ocean World technology development and analog testing but, in the future, will only be selected for competition in alternate years, adding pressure to a proven but already extremely competitive program. PSTAR and its precursor, ASTEP, have a strong history of funding work wherein various Antarctic 
sites have been used as analog environments for NASA astrobiology research and technology development. Beginning in 2017, however, PSTAR requests for proposals have explicitly stated that work in the Antarctic will not be considered for funding, essentially cutting off all ability to test technology in this important analog. Regardless of the reasons and history behind the current state of affairs, steps must be taken to repair and reopen active and collegiate cooperation and collaboration between these agencies. For Ocean World Life-Search Missions to become a reality and move smartly forward, action is needed at a high level to call attention to the importance of improved cooperation between NASA and NSF in the Antarctic. Both NASA and NSF have responsibilities to reach this goal for our nation.

\section{Concluding Remarks}

We present a critical need for two distinct testing environments to ensure that the cryobots representing significant technological breakthroughs for the exploration of Ocean Worlds can be rigorously tested prior to mission deployment. Four of the six elements of a sub-surface Ocean World mission can be most effectively tested at Ocean Worlds Field Station. The 4,000 meters of ice over sub-glacial lakes in Antarctica represent the last 4 kilometers of ice before breakthrough to an icy moon ocean. The logistical costs of establishing, operating, and maintaining the test site will be small compared to the cost of conducting the actual flight missions and the fidelity of the environment at Ocean Worlds Field Station for truly testing fully-integrated systems for long duration missions will be superior to anything that could be attempted in the laboratory. Long-duration missions at Ocean Worlds Field Station will enable the rapid iteration of flight and subsystem designs. Conversely, the Starting Problem and Cold Cruise - Elements 1) and 2) above - can only be addressed in a laboratory test environment, and so we recommend the establishment of a national cryogenic-ice vacuum test facility - Ocean World Simulator Station - leveraging community experience gained from smaller-scale facility development and operation.

[1] †Howell+(2020). Ocean Worlds Exploration and the Search for Life, arXiv:2006.15803; [2] $\uparrow$ Schmidt+ (2020) Subsurface Needs for Ocean Worlds (SNOW sub-committee of OPAG); [3] Stone+ (2018) Project VALKYRIE: Laser-powered Cryobots and Other Methods for Penetrating Deep Ice on Ocean Worlds, doi.org/10.1007/978-3-319-73845-1; [4] Zacny+(2020) Advances in Terrestrial and Extraterrestrial Drilling: Ground, Ice, and Underwater. Forthcoming, CRC Press/ Taylor \& Francis Group LLC; [5] Stone+ (2014) Progress towards an optically powered cryobot," Annals of Glaciology, 55(66) DOI: 10.3189/2014AoG65A200; [6] Dachwald+ (2020) Key Technologies and Instrumentation for Subsurface Exploration of Ocean Worlds. Space Sci Rev 216, 83 (2020). doi.org/10.1007/s11214-020-00707-5; [7] Barker+ (2020) Scientific challenges and present capabilities in underwater robotic vehicle design and navigation for oceanographic exploration under-ice, doi: 10.3390/rs12162588, 2020; [8] Winebrenner+ (2013) A Thermal Ice-Melt Probe for Exploration of Earth-Analogs to Mars, Europa and Enceladus, Lunar and Planetary Science Conference. Vol. 44; [9] Zacny+ (2018), IEEE Aerospace Conference, 1-14; [10] Stone+ (2018) Sub-Ice Autonomous Underwater Vehicle Architectures for Ocean World Exploration and Life Search, doi.org/10.1007/978-3-319-73845-1; [11] Howell, S., R.T. Pappalardo (2020), NASA's Europa Clipper - a mission to a potentially habitable ocean world? Nature Communications, doi:10.1038/s41467-020-15160-9| †Denotes Decadal White Paper. 